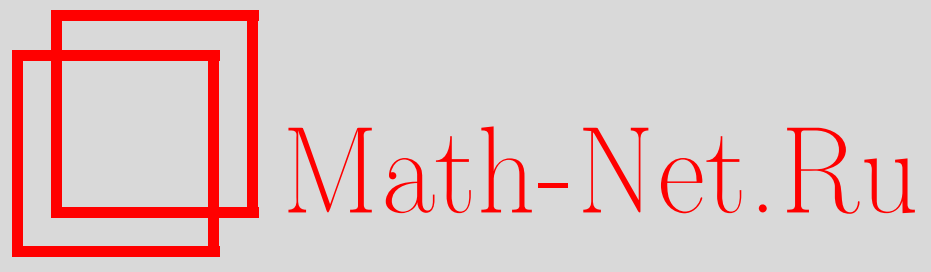

Н. П. Долбилин, Теоремы Минковского о параллелоэдpax и их обобщения, УМH, 2007, том 62, выпуск 4, 157158

DOI: https://doi.org/10.4213/rm6813

Использование Общероссийского математического портала Math-Net.Ru подразумевает, что вы прочитали и согласны с пользовательским соглашением http://www . mathnet.ru/rus/agreement

Параметры загрузки:

IP : 18.234 .156 .22

26 апреля 2023 г., $17: 27: 50$

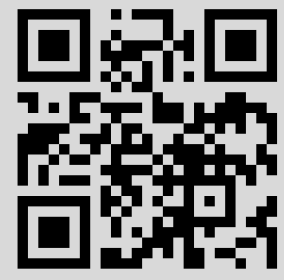




\section{Теоремы Минковского о параллелоэдрах и их обобщения}

\section{Н. П. Долбилин}

Параллелоэдр определяется как выпуклый $d$-мерный многогранник, нормально (т. е. грань-в-грань) разбивающий евклидово пространство $\mathbf{E}^{d}$ параллельными копиями. Минковский доказал две фундаментальные теоремы (см. [1], [2]).

Теорема 1 (Минковский). Если $P-d$-мерный параллелоэдр, то

1) $Р$ иентрально-симметричен;

2) все гиперграни многогранника $P$ чентрально-симметричны;

3) проекция $P$ вдоль любой его $(d-2)$-грани на дополнительную 2-плоскость есть либо параллелограмм, либо центрально-симметричный шестиугольник.

Теорема 2 (Минковский). Число $f_{d-1}(P)$ гиперграней у $d$-параллелоэдра $P$ не превышает $2\left(2^{d}-1\right)$; причем существует параллелоэдр $P$ с $f_{d-1}=2\left(2^{d}-1\right)$.

В заметке уточняются обе теоремы, и показывается, что они верны и в более общем случае, когда разбиение пространства на параллельные друг другу выпуклые многогранники не предполагается нормальным. Пусть $T$ - нормальное разбиение на параллелоэдры и $P, P^{\prime} \in T$ - такие, что $P \cap P^{\prime} \neq \varnothing$. Пересечение $P \cap P^{\prime}$ является гранью $F^{i}$ некоторой размерности $i$. Грань $F^{i}$, являющуюся пересечением $P \cap P^{\prime}$ двух параллелоэдров, будем называть максимальной общей гранью для $P$ и $P^{\prime}$ (М.О.Г.). Подчеркнем, что не всякая грань параллелоэдра является М.О.Г. Например, в разбиении плоскости на правильные шестиугольные соты никакая вершина не является М.О.Г. (размерности 0), в то время как все стороны ячейки являются М.О.Г. (размерности 1).

Особо отметим, что для параллелоэдра каждая его гипергрань $F^{d-1}$ является пересечением двух и только двух параллелоэдров и поэтому является М.О.Г.

Примеры параллелоэдров и их максимальных общих граней.

1. Параллелепипед. Все $i$-грани, $0 \leqslant i \leqslant d-1$, - максимальные общие.

2. Примитивный $d$-параллелоэдр - это, по определению, такой параллелоэдр, что в каждой вершине соответствующего разбиения сходится минимально возможное число (а именно $d+1)$ параллелоэдров. Легко видеть, что примитивный параллелоэдр является простым многогранником. Поэтому каждый параллелоэдр $P^{\prime}$, имеющий с $P$ непустое пересечение, имеет с $P$ общую гипергрань. В примитивном параллелоэдре никакая грань размерности меньше $d-1$ не является максимальной общей.

Из центральной симметричности параллелоэдра (теорема 1, п. 1)) легко следует

Утверждение 1. Каждая максималъная общая грань F параллелоэдра является центрально-симметричной гранъю; иентр симметрии грани $F$ является иентром симметрии разбиения. Обратно, любой центр симметрии разбиения является иентром симметрии некоторой М.О.Г. параллелоэдра разбиения.

Так как любая гипергрань является максимальной общей, то утверждение 1 является усилением п. 2) теоремы 1 Минковского. Из утверждения 1 следует

УтвеРЖДЕнИЕ 2. В разбиении для каждой максимальной общей грани $F$ параллелоэдра $P$ существует лищь один параллелоэдр $P^{\prime}$ такой, что $F=P \cap P^{\prime}$.

Параллелоэдры, сходящиеся в грани $F$, образуют звезду $\operatorname{St}(F)$ грани $F$.

УтвеРЖДЕниЕ 3. Для максимальной общей грани $F$ звезда $\operatorname{St}(F)$ центрально симметрична. Более того, для любого параллелоэдра $P \in \operatorname{St}(F)$ имеется один и только один параллелоэдр $P^{\prime} \in \mathrm{St}(F)$ такой, что $P \cap P^{\prime}=F$.

УтвержДЕниЕ 4. Если $F$ - не максимальная общая гранъ, то для параллелоэдров $P$ и $P^{\prime} \in \operatorname{St}(F)$ грань $F$ является подгранъю их общей грани: $F \subset P \cap P^{\prime}$.

Работа выполнена при поддержке РФФИ (гранты 05-01-00170 и 06-01-72551). 
Валентностъю $n(F)$ грани $F$ параллелоэдра назовем число параллелоэдров в $\operatorname{St}(F)$. Напомним, что $i$-грань $F^{i}$, где $0 \leqslant i \leqslant d-2$, называется примитивной, если ее валентность минимальна: $n\left(F^{i}\right)=d+1-i$. Нетрудно вывести, что если грань $F^{i}$ примитивна, то примитивна и любая ее надгрань $F^{j}, F^{i} \supset F^{j}, j>i$. В частности, в примитивном параллелоэдре грани всех размерностей примитивны. Примитивность $(d-2)$-грани означает, что $n\left(F^{d-2}\right)=3$. Из утверждений 3 и 4 немедленно следует

УтвеРЖДЕниЕ 5. Валентность любой $(d-2)$-грани $F^{d-2}$ параллелоэдра либо 3 , либо 4. Если $n\left(F^{d-2}\right)=4$, то $F^{d-2}-$ М.О.Г.

Из утверждения 5 вытекает свойство 3) теоремы 1 Минковского.

ГипотезА. Пусть $F^{k}-$ М.О.Г., тогда общее число всех граней всех размерностей в $\mathrm{St}\left(F^{k}\right)$ в точности равно $3^{d-k}$.

Гипотеза верна для $k=d-2$ (утверждение 5$)$ и для $k=d-3$.

Индексом грани $F$ назовем число $\nu(F):=1 / n(F)$. Обозначим через $\mathscr{M}$ множество всех М.О.Г. в параллелоэдре $P$.

Теорема. Для параллелоэдра выполнено равенство

$$
\sum_{F \in \mathscr{M}} \nu(F)=2^{d}-1
$$

ИДЕЯ ДОКАЗАТЕЛЬСТВА. Пусть $\Lambda$ - целочисленная решетка центров параллелоэдров. $\Lambda$ распадается в $2^{d}$ классов: $\Lambda:=\Lambda_{0} \cup \Lambda_{1} \cup \cdots \cup \Lambda_{2^{d}-1}$. Здесь $\Lambda_{0}$ состоит из точек $\lambda \in \Lambda$, у которых все координаты в базисе решетки четные. Каждому 0/1-вектору $\mu_{i}=\left(m_{1}^{i}, \ldots, m_{d}^{i}\right) \in \Lambda$, соответствует класс $\Lambda_{i}=\left\{\lambda \in \Lambda \mid \lambda \equiv \mu_{i}(\bmod 2)\right\}=\Lambda_{0}+\mu_{i}$. Множество середин всех отрезков с концами в точках решетки $\Lambda$ образует решетку $\Gamma=\frac{1}{2} \Lambda$. Фактор $\Gamma / \Lambda$ состоит из $2^{d}$ классов: $\Gamma / \Lambda=\left\{\Gamma_{i} \mid i=0,1, \ldots, 2^{d}-1\right\}$, где $\Gamma_{i}=\Lambda_{0}+\frac{1}{2} \mu_{i}$. Поэтому для $\gamma_{i}$ и $\gamma_{i}^{\prime} \in \Gamma_{i}$ имеем $\gamma_{i}-\gamma_{i}^{\prime} \in \Lambda, \Gamma_{0}=\Lambda$

Пусть $\gamma \in \Gamma$ и $s_{\gamma}$ - симметрия относительно $\gamma$, переводящая центр $O \in P_{O}$ в центр $O^{\prime} \in P_{O^{\prime}}$. Тогда $P_{O} s_{\gamma}=P_{O^{\prime}}$. Ясно, что $P_{O} \cap \Lambda=\operatorname{Int}\left(P_{O}\right) \cap \Gamma=\{O\}$. Действительно, $\partial P_{O} \cap \Gamma_{i} \neq \varnothing$ для любого $i \neq 0$, где $\partial P_{O}-$ граница $P_{O}$. Пусть точка $\gamma_{i} \in \partial P_{O}$ принадлежит также еще $n_{i}-1$ параллелоэдрам $P_{O_{1}}, \ldots, P_{O_{n_{i}-1}}$. Тогда $\Gamma_{i} \cap \partial P_{O}=\left\{\gamma_{i}, \gamma_{i}+\overrightarrow{O_{1} O}, \ldots, \gamma_{i}+\overrightarrow{O_{n_{i}-1} O}\right\}$. Следовательно, каждый класс $\Gamma_{i}$ имеет на границе $\partial P_{O}$ в точности $n_{i}$ представителей. С другой стороны, каждой точке из $(\Gamma \backslash \Lambda) \cap \partial P_{O}$ соответствует М.О.Г. $F$ некоторой размерности, причем ее индекс $n(F)$ равен $n_{i}$. Обратно, каждой М.О.Г. $F \subset \partial P$ соответствует точка $\lambda_{i}$ с $n_{i}=n(F)$. Поэтому в сумме $(1)$ каждое слагаемое $1 / n(F)$ соответствует одному и только одному классу $\Gamma_{i}, 1 \leqslant i \leqslant 2^{d}-1$, откуда следует (1).

Так как индекс гиперграни равен $1 / 2$, то из (1) следует, что количество гиперграней не превышает $2\left(2^{d}-1\right)$. Более того, $f_{d-1}=2\left(2^{d}-1\right)$ тогда и только тогда, когда у параллелоэдра других М.О.Г. кроме гиперграней нет.

Теорема 1 Минковского верна для выпуклых многогранников, которые разбивают пространство параллельными копиями не обязательно нормально. Действительно, так как многогранники параллельны друг другу, то каждая гипергрань многогранника $P$ должна иметь себе параллельную гипергрань. Доказывается, что параллельные гиперграни имеют одинаковый $(d-1)$-объем. Поэтому многогранник центрально симметричен. Пункт 1) теоремы 1 установлен. Доказательство п. 2) опирается на то, что если выпуклый многогранник является объединением попарно неперекрывающихся центрально симметричных многогранников, то он сам центрально-симметричен.

\section{Список литературы}

[1] H. Minkowski, Nachr. Ges. Wiss. Göttingen, 1897, 198-219. [2] А. Д. Александров, Въпуклые многогранники, Гостехиздат, М.-Л., 1950.

Н. П. Долбилин (N.P. Dolbilin)

Математический институт им. В. А. Стеклова РАН

E-mail: dolbilin@mi.ras.ru
Представлено В. М. Бухштабером Принято редколлегией 06.04.2007 